\title{
Fatal outcome of renal transplantation in a patient with the Wiskott-Aldrich syndrome
}

\author{
A. Fischer ${ }^{1}$, I. Binet ${ }^{1}$, D. Oertli ${ }^{2}$, A. Bock ${ }^{1}$ and G. Thiel ${ }^{1}$ \\ ${ }^{1}$ Division of Nephrology, Department of Internal Medicine, and 2 Department of Surgery, Kantonsspital, University of Basel, \\ Basel, Switzerland
}

Key words: B-cell lymphoma; renal transplantation; Wiskott-Aldrich syndrome

\section{Introduction}

Two cases of successful cadaveric renal transplantation in patients with the Wiskott-Aldrich syndrome have previously been reported in the literature $[1,2]$. The first patient [1] had an almost uneventful outcome, while the second one [2] had to be treated with highdose methylprednisolone for biopsy-proven cellular rejection. The optimal immunosuppressive regimen in patients with Wiskott-Aldrich syndrome remains unknown. We report here the case of a Wiskott-Aldrich patient who underwent living-related renal transplantation. This patient however developed a number of infectious and non-infectious complications which led to his death 3 months after transplantation.

\section{Case report}

The patient, a 41-year-old man with a strong family history of an X-linked transmitted syndrome (17 affected men and 10 female carriers in the last three generations), presented in early childhood with severe thrombocytopenia, allergic eczema, and recurrent infections (bronchitis, herpetic keratitis). Since 1982 he had developed progressive renal insufficiency with microhaematuria and proteinuria, which was presumed to be due to I gA nephropathy (no biopsy was obtained, but a brother of the patient who died at the age of 32 suffered from the Wiskott-Aldrich syndrome and developed end-stage renal failure due to biopsyproven I gA nephropathy). Haemodialysis treatment was started in April 1991. In preparation for renal transplantation the patient underwent splenectomy in

Correspondence and offprint requests to: Prof. Gilbert Thiel, Division of Nephrology, Department of Internal Medicine, Kantonsspital, Petersgraben 4, CH-4031 Basel, Switzerland.
January 1994, which resulted in normalization of the platelet count.

In August 1994 he received a one-haplotype-matched living-related kidney graft (the donor was his father born in 1925). CMV constellation was donor positive/ recipient positive. Epstein-Barr virus serology was I gG positive and I gM negative for both recipient and donor. Because of the underlying immunosuppression, our usual induction protocol (quadruple therapy including antithymocyte globulin (ATG), azathioprine (Aza), prednisone (Pred), and CsA was modified by omitting ATG. CsA (dose adjusted to maintain a trough whole-blood level of $200-250 \mathrm{ng} / \mathrm{ml}$ ), Aza $(2 \mathrm{mg} / \mathrm{kg})$, and methylprednisolone $(1 \mathrm{~g}, 0.5 \mathrm{~g}, 0.25 \mathrm{~g}$ intravenously respectively on day $0,1,2)$ tapered to $0.5 \mathrm{mg} / \mathrm{kg}$ Pred by day 3 were administered as usual. The onset of renal function was prompt. On day 5 creatinine increased and three methylprednisolone pulses were administered. Transplant biopsy failed to confirm rejection and functional CsA toxicity was suspected. Creatinine decreased $(151 \mu \mathrm{mol} / \mathrm{l}$ on day 14). On day 22 creatinine increased to $229 \mu \mathrm{mol} / \mathrm{l}$ and a second renal biopsy showed a moderate infiltrative and proliferative transplant vasculopathy with areas of arteriolar necrosis. ATG $(0.2 \mathrm{ml} / \mathrm{kg}$ per day) was administered for 7 days with a good effect on renal function.

On day 30 the patient developed CMV disease and was treated with ganciclovir. Because of severe leukopenia filgrastim was administered over 5 days. On day 39 a catheter sepsis with coagulase-negative staphylococci was diagnosed and treated with vancomycin. On day 46, several herpes zoster vesicles appeared on the plantar edge of the left foot. On day 52 the patient developed fever and paroxysmal excruciating pain radiating to the left lower limb with a S1 dermatome topography. Acyclovir was administered and clonazepam was necessary to control pain, which was interpreted as zoster neuralgia. From days 76 to 78 the patient was given three methylprednisolone pulses for biopsy-proven interstitial rejection (creatinine $237 \mu \mathrm{mol} / 1$.).

High fever appeared associated with rising liver 
enzymes (ASAT up to $858 \mathrm{U} / \mathrm{l}$, ALAT up to $369 \mathrm{U} / 1$ ). Ganciclovir and imipenem were given but without improvement of the patient's condition. A liver biopsy on day 88 revealed a malignant high-grade B-cell nonHodgkin lymphoma (large-cell immunoblastic according to International Working Formulation) with massive periportal infiltration. A thoracoabdominal CT scan and bone marrow aspiration failed to demonstrate any other foci of the lymphoma. CsA was stopped on day 88 and a first cycle of chemotherapy consisting of adriamycin and cyclophosphamide was administered. On the same day, Pneumocystis carinii pneumonia was diagnosed and treatment with co-trimoxazole was instituted. Haemodialysis was then started because of progressive oliguria. On day 91 the patient developed massive epistaxis and melena requiring transfusional support; platelets had decreased to $38000 / \mathrm{mm}^{3}$. Two days later a subdural haematoma was diagnosed by CT scan.

Over the following days the patient's condition rapidly deteriorated. He became unresponsive, developed severe hypotension, and died on day 98. Autopsy was not performed because of family refusal. Post-mortem liver biopsy showed persistent lymphoma. Post-mortem transplant biopsy showed mild sclerotic transplant vasculopathy.

\section{Discussion}

The Wiskott-Aldrich syndrome is a rare $\mathrm{X}$-linked syndrome associating B- and T-cell immunodeficiency, thrombocytopenia, and eczema. As recently described $[3,4]$ there is a defective expression of the CD43 molecule on $\mathrm{T}$ cells, which may compromise the antigen specific activation of $\mathrm{T}$ cells by impairing interaction with ICAM-1. This same mechanism may underlie the propensity for infections and the increased incidence of B-cell lymphoma for which this syndrome is known [5]. The ideal treatment for patients with the Wiskott-Aldrich syndrome is HLA-identical bonemarrow transplantation from a sibling donor, which cures the underlying immunodeficiency, although such donors are available for only a few Wiskott-Aldrich patients $[6,7]$. An alternative therapeutic approach is splenectomy, which has been shown to improve both survival and quality of life [7].

One variant of the syndrome is associated with I gA nephropathy, potentially leading to end-stage renal failure [8-10]. Renal transplantation is not usually recommended in this condition because of the underlying immunodeficiency and the increased risk of lymphoma. The patient reported by Webb et al. [1] was given a reduced immunosuppressive regimen (triple therapy with Aza in a reduced dose, CsA, and Pred). There was no rejection episode and a primary CMV seroconversion illness resolved rapidly after treatment with anti-CMV hyperimmune globulins. A very similar immunosuppressive regimen was initially used by Meisels et al. [2]; Aza had to be discontinued on day 12 because of thrombocytopenia. Their patient, however, had to be treated twice with high-dose methylprednisolone, the first time for clinically suspected rejection, the second time for biopsy-proven cellular rejection. This shows that despite the underlying defect in cellular immunity their patient was able to develop an immune response to allograft antigens. Our patient underwent a one-haplotype-matched living-related renal transplantation. He received two courses of high dose methylprednisolone, and was additionally treated with ATG because of vascular rejection. The following course proceeded to the dreaded 'worst-case scenario': several infectious complications followed each other in rapid succession and a fatal high grade B-cell lymphoma of the liver developed.

Such a dramatic outcome raises ethical as well as medical considerations. Our observation suggests that renal transplantation remains a high-risk procedure in patients with the Wiskott-Aldrich syndrome. These patients are able to mount a severe rejection reaction against the transplanted kidney and their increased propensity to develop lymphoma limits drastically the therapeutic possibilities if such a rejection occurs. Retrospectively it is evident that we superseded this limitation. We probably should have reduced the amount of Aza in our induction protocol, and certainly should not have used ATG at all. The patient was a perfectly well-informed man working in a leading paramedical position. Every step in his treatment was thoroughly discussed and our error was to accept his willingness to undergo a risky treatment. Is it at all ethical to transplant a patient with the Wiskott-Aldrich syndrome? The answer to this question depends probably most on the subjective quality of life during chronic dialysis treatment. If renal transplantation is attempted, Aza dose is to be reduced and antilymphocyte globulins are to be strictly avoided, even if this means rapidly losing the graft.

\section{References}

1. Webb MC, Andrews PA, Koffman CG, Cameron JS. Renal transplantation in Wiskott-Aldrich syndrome. Transplantation 1993; $56: 747-748$

2. Meisels IS, Strom TB, Roy-Chaudhury $P$ et al. Renal allograft rejection in a patient with the Wiskott-Aldrich syndrome Transplantation 1995; 59: 1214-1215

3. Park JK, Rosenstein YJ, Remold-O'Donnel E, Bierer BE, Rosen FS, Burakoff SJ. Enhancement of T-cell activation by the CD 43 molecule whose expression is defective in Wiskott-Aldrich syndrome. Nature 1991; 350: 706-709

4. Rosenstein YJ, Park JK, Hahn WC, Rosen FS, Bıerer BE, Burakoff SJ. CD 43, a molecule defective in Wiskott-Aldrich syndrome binds ICAM-1. Nature 1991; 354: 233-235

5. Cotelingam JD, Witebsky FG, Hsu SM, Blaese RM, Jaffe ES. Malignant lymphoma in patients with the Wiskott-Aldrich syndrome. Cancer Invest 1985; 3: 515-522

6. Rimm IJ, Rappeport JM. Bone marrow transplantation for the Wiskott-Aldrich syndrome. Long-term follow-up. Transplantation 1990; 50: 617-620

7. Mullen CA, Anderson KD, Blaese M. Splenectomy and/or bone marrow transplantation in the management of the Wiskott-Aldrich syndrome: long-term follow-up of 62 cases. Blood 1993; 82: 2961-2966 
8. Guttenberger J, Trygstad CW, Stiehm ER et al. Familial thrombocytopenia, elevated serum I gA levels and renal disease. A report of kindred. $A m J$ Med 1970; 49: 729-741

9. Standen GR, Lillicrap DP, Mathews N, Bloom AL. Inherited thrombocytopenia, elevated serum I gA and renal disease. Identification of a variant of the Wiskott-Aldrich syndrome. $Q$ $J$ Med 1986; 59: 401-408

10. Spittler LE, Wray BB, Morgenmanns S, Mıller JJ, O'Reilly RJ, Lagios $M$. Nephropathy in the Wiskott-Aldrich syndrome. Pediatrics 1980; 66: 391-398
Received for publication 1.12 .95

Accepted in revised form. 8.1295

\section{Editor's note}

Please see also The Interesting Case by Hitzig and Triniger (pp. 2093-2095 in this issue). 